\title{
El género Gomphocarpus (Apocynaceae: Asclepiadoideae) en la Argentina
}

\author{
The genus Gomphocarpus (Apocynaceae: Asclepiadoideae) in Argentina
}

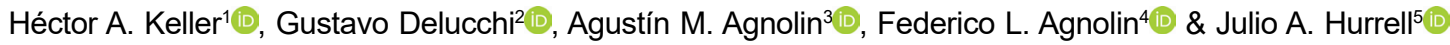

\begin{abstract}
Resumen: Se reporta la presencia de dos especies del género Gomphocarpus (Apocynaceae, Asclepiadoideae) que crecen espontáneamente en la Argentina: G. fruticosus (escapada de cultivo) y G. physocarpus (naturalizada). Las mismas se describen, se indica su distribución geográfica, usos, polinización y dispersión. Además, se analiza el estatus de naturalización de estas especies en el país.
\end{abstract}

Palabras clave: Dispersión, Gomphocarpus, naturalización, polinización.

\begin{abstract}
Summary: The presence of two species of Gomphocarpus (Apocynaceae, Asclepiadoideae) that grow spontaneously in Argentina is reported here: G. fruticosus (cultivation escaped) and $G$. physocarpus (naturalized). Both species are described, and their geographical distribution, uses, pollination and dispersal are indicated. In addition, the naturalization status of these species in the country is analyzed.
\end{abstract}

Key words: Dispersal, Gomphocarpus, naturalization, pollination.

\section{Introducción}

Como parte de diversos estudios sobre especies escapadas de cultivo, naturalizadas y potenciales invasoras de interés etnobotánico introducidas en la Argentina, se constató la presencia de dos especies de Gomphocarpus (Apocynaceae, Asclepiadoideae). Se trata de G. fruticosus (L.) W. T. Aiton, hallada en las provincias de Corrientes y San Juan y G. physocarpus E. Mey., coleccionada en las provincias de Misiones, Entre Ríos y Buenos Aires, y en la Ciudad Autónoma de Buenos Aires. Ambas especies han sido introducidas en nuestro país debido a su valor ornamental (Fabris, 1966; Dimitri, 1988; Hurrell et al., 2004), y participan del proceso de naturalización de especies exóticas (Richardson et al., 2000, 2011; Pyšek et Richardson, 2006).

Por lo general, las especies de Gomphocarpus son pioneras en sitios abiertos, en bordes de sendas y caminos, y en ambientes perturbados; en particular, G. physocarpus

${ }^{1}$ Facultad de Ciencias Forestales, Universidad Nacional de Misiones, Instituto de Botánica del Nordeste, Casilla de Correo 209, 3400-Corrientes. Investigador CONICET, Argentina. E-mail: kellerhector@hotmail.com

${ }^{2}$ Facultad de Ciencias Agrarias y Forestales, 60 y 119 y Facultad de Ciencias Naturales y Museo, Paseo del Bosque s/n, Universidad Nacional de La Plata (1900). La Plata, Argentina.

${ }^{3}$ Instituto Nacional de Antropología y Pensamiento Latinoamericano, 3 de Febrero 1378, C1426BJN, Buenos Aires, Argentina. CONICET.

${ }^{4}$ Laboratorio de Anatomía Comparada y Evolución de los Vertebrados, Museo Argentino de Ciencias Naturales "Bernardino Rivadavia". Ángel Gallardo 470, C1405BDB, Buenos Aires, Argentina. CONICET. Fundación de Historia Natural "Félix de Azara". Universidad Maimónides, Hidalgo 775, C1405BDB, Buenos Aires, Argentina.

${ }^{5}$ Laboratorio de Etnobotánica y Botánica Aplicada, Facultad de Ciencias Naturales y Museo, Universidad Nacional de La Plata. Calle 64 nro. 3, 1900, La Plata, Argentina. 
que se ha naturalizado y devenido invasora y/o maleza en distintas partes del mundo (Goyder et Nicholas, 2001). El proceso de naturalización en las especies de este género se ve favorecido por la dispersión anemócora de sus semillas con pelos sedosos y un sistema de polinización generalista y flexible realizado por avispas (Hymenoptera: Vespidae) similares en diferentes zonas del planeta, que contribuyen al éxito de la colonización de nuevos ambientes (Coombs et al., 2009).

\section{Resultados}

Gomphocarpus R. Br., Asclepiadeae: 26. 1810. Especie tipo: Gomphocarpus fruticosus (L.) W. T. Aiton, Hort. Kew (ed. 2) 2: 80. 1811.

Gomphocarpus secc. Eugomphocarpus Decne., Prodr. (DC.) 8: 557. 1844.

Gomphocarpus subsecc. Leiocalymma K. Schum., Nat. Planzenfam. 4(2): 236. 1895.

Arbustos o arbolitos con tallos erectos, ramificados desde la base o en la parte superior. Hojas opuestas, subopuestas o pseudoverticiladas; pecíolos cortos, láminas oblongas, lineares, linear-elípticas, elípticas, ápice agudo o atenuado, base cuneada. Inflorescencias extraaxilares umbeliformes, pedunculadas. Flores pediceladas; cáliz profundamente partido; corola blanca, tubo corto, lóbulos fuertemente reflejos; corona ginostegial blanca o lilácea, con cinco lóbulos interestaminales diminutos y cinco lóbulos estaminales cuculados, bien desarrollados, margen superior con un diente a cada lado. Anteras con alas conspicuas. Cabeza estilar pentalobulada, aplanada o algo cóncava, exerta o parcialmente incluida en los lóbulos de la corona. Polinios colgantes, aplanados; retináculo obovoide, mucho más pequeño que los polinios, caudículas planas, ensanchadas en la unión con las polinias. Frutos globosos u ovoides, en general inflados, ápice atenuado, rostrado, o redondeado, sin rostro, superficie ornamentada con apéndices filiformes. Semillas comosas, ovoides, con la cara dorsal cóncava y la cara ventral convexo-marginada, superficie verrucosa, ápice redondeado.
Género con unas 20 especies nativas de regiones secas de África y áreas contiguas de Arabia, el Sinaí, Israel y Jordania. Se caracteriza por sus raíces fibrosas, sus tallos ramificados e inflorescencias extraaxilares, a diferencia del género afín Asclepias L., que presenta cáudices subterráneos tuberosos, napiformes $\mathrm{y}$, por lo general, tallos no ramificados e inflorescencias terminales $(\mathrm{Li}$ et al., 1995; Goyder et Nicholas, 2001). En la Argentina crecen de forma espontánea dos especies en diferentes etapas del proceso de naturalización (Richardson et al., 2000, 2011; Pyšek et Richardson, 2006).

1. Gomphocarpus fruticosus (L.) W. T. Aiton, Hort. Kew (ed. 2) 2: 80. 1811. Asclepias fruticosa L., Sp. P1. 1: 216. 1753. Asclepias salicifolia Salisb., Prodr. Stirp. Chap. Allerton 150. 1796. Typus: Sin datos de localidad ni fecha, Anónimo (Lectotypus LINN-HL310-33!, designado por Wijnands, Bot. Commelins 48. 1983). South Africa. Northern Cape, Bloeddrif, 12 Sep 1968, Hardy 2562 (Epitypus, K designado por D. J. Goyder \& A. Nicholas, Kew Bull. 56: 782. 2001, isoepitypus, PRE, WIND).

Asclepias glabra Mill., Gard. Dict. (ed. 8): $\mathrm{n}^{\mathrm{0}}$ 12. 1768. Typus: 'Asclepias glabra Mill., Gard. Dict. $n^{o} 12^{\prime}$ (Holotypus, BM).

Iconografia: Fabris, 1966: 76; Goyder et Nicholas, 2001: fig. 2 A.

Arbustos de 0,5-3 m de alt., ramificados desde la base, rámulos pubescentes, raro tomentosos. Hojas opuestas; pecíolo de 1-10 mm de long.; lámina linear a linear-elíptica, de 2,5-12 $\times$ 0,2-1,3 cm, ápice agudo a atenuado, base estrecha a anchamente cuneada, subglabras, pubescencia esparcida, con tricomas suaves en la vena media y el margen. Inflorescencias con 4-12 flores; pedúnculo de 1,5-4 cm de long., pubescente; brácteas filiformes, deciduas; pedicelo de 1-2,5 cm de long., pubescente; cáliz con lóbulos de 2-5 × 0,6-1,3 mm, lanceolados o triangular-atenuados, pubescentes; corola blanca, refleja, lóbulos de 5-8 × 3-5 mm, ovados, subagudos, cara adaxial minutamente papilosa y margen derecho densamente cubierto de largos tricomas blancos; corona 
con lóbulos blancos o liláceos, unidos a la columna estaminal a 1-1,5 mm sobre la base de esta, lateralmente comprimidos, de 2-4 $\times$ 1,5-3 mm, tan altos como la columna, margen superior entero; cabeza estilar plana; anteras con alas de 1,5-2 mm de long.; retináculo de $0,3 \times 0,10-0,15 \mathrm{~mm}$, subcilíndrico; caudículas ca. 0,3 mm de long.; polinios de 1,2-1,3 $\times$ $0,3 \mathrm{~mm}$, aplanados, de contorno oblongo $\mathrm{u}$ oblanceolado. Frutos folículos con pedúnculos contortos, erectos, ovoides, de 4-7 × 1,5$2,5 \mathrm{~cm}$, inflados, pubescentes, ápice agudo, rostrado; cubiertos con apéndices filiformes ca. $1 \mathrm{~cm}$ de largo. Semillas obovoides, comosas, de 3,5-5 $\times 2 \mathrm{~mm}$, superficie verrucosa.

Distribución geográfica y hábitat: Especie nativa del sur de África: Angola, Namibia, Zambia, Malawi, Mozambique, Botsuana, Zimbabue, Sudáfrica, Suazilandia y Lesoto. Crece en suelos arenosos, secos, en ambientes abiertos y sitios perturbados, con frecuencia en bordes de cursos de agua. Se halla naturalizada en zonas templado-cálidas y áreas subtropicales secas del mundo, en muchos sitios deviene maleza y/o invasora (Goyder et Nicholas, 2001; Randall, 2017). En la Argentina crece de forma espontánea escapada de cultivo en Corrientes y en San Juan. Para el ejemplar de Corrientes (Pedersen 13493, CTES) se señalan renuevos espontáneos a partir de los cultivos, que no llegan a naturalizarse (Dematteis et Ferrucci, 2017).

Fenología, polinización, dispersión: Florece $\mathrm{y}$ fructifica en verano. La polinización es entomófila (principalmente por véspidos). Las semillas con pelos sedosos tienen dispersión anemócora (Goyder et Nicholas, 2001).

Nombres vernáculos: "Bolas de fraile", "globitos", "planta de la seda" (Argentina).

Usos: Se cultiva como ornamental para parques y jardines (Fabris, 1966; Dimitri, 1988). Se reproduce por semillas y tiene crecimiento rápido; requiere suelos sueltos, bien drenados. En medicina popular, la decocción de la planta se emplea para tratar las dolencias estomacales; las hojas, para combatir la tuberculosis pulmonar. Los pelos sedosos de las semillas se emplean para elaborar almohadas (Li et al., 1995). Gomphocarpus fruticosus contiene glucósidos flavonoides tóxicos que le confieren una defensa química muy efectiva frente a herbívoros generalistas silvestres y frente al ganado (Fernández Haeger et Jordano Barbudo, 2009).

Material estudiado: ARGENTINA. Corrientes: Dep. Mburucuyá, Parque Nacional Mburucuyá, 27-1-1983, Pedersen 13493 (CTES). San Juan: Dep. Zonda, Barreal, 11-II1986, Kiesling et al. 6100 (SI).

2. Gomphocarpus physocarpus E. Mey., Comm. P1. Afr. Austr. 1(2): 202. 1838. Asclepias physocarpa (E. Mey.) Schltr., Bot. Jahrb. Syst. 21 (Beibl. 54): 8. 1896. Typus: Sudáfrica, by stream near Glenfilling, $A$. Drege s.n. (Lectotypus K (Herbario Benthamianum), designado por D. J. Goyder, Kew Bull. 53: 418. 1998; isolectotypus BM, E, K, TCD).

Gomphocarpus brasiliensis E. Fourn., Fl. Bras. 6(4): 203, tab. 53. 1885. Gomphocarpus fruticosus (L.) R. Br. fo. brasiliensis (E. Fourn.) Briq., Kongl. Svenska Vetenkapsakad. Handl., n.s. 34(7): 453. 1896. Asclepias brasiliensis (E. Fourn.) Schltr., Meded. Rijks-Herb. 29: 12. 1916. Typus: Brasil, towards Petropoli, 8 Sep 1873, A. F. M. Glaziou 6706 (Lectotypus, P00642569!, designado por D. J. Goyder \& A. Nicholas, Kew Bull. 56: 782. 2001; isolectotypi C10006698!, K00096474!).

Iconografia: Fournier, 1885 (loc. cit.); Goyder et Nicholas, 2001: fig. 2C; Hurrell et al., 2004: 95.

Arbustos o arbolitos erectos de 1-2,5 m de alto, tallos ramificados hacia el ápice, rámulos pubescentes. Hojas opuestas, rara vez subopuestas o pseudoverticiladas; pecíolo de 3-12 mm de long.; lámina estrechamente oblonga a elíptica, de $4-12 \times 0,5-2 \mathrm{~cm}$, ápice agudo, base cuneada, subglabras, pubescencia esparcida, con tricomas blancos. Inflorescencias con 4-12 flores; pedúnculo de 1,5-3,5 cm de long., ascendente, pubescente a tomentoso; brácteas deciduas; pedicelo de 1-3 cm de long., densamente pubescente; cáliz con lóbulos de 3-4 $\times 1 \mathrm{~mm}$, estrechamente triangulares, 
pubescentes; corola blanca, fuertemente refleja, lóbulos ovados, de 5-8 × 3-4,5 mm, subagudos, cara adaxial minutamente papilosa y con el margen derecho densamente cubierto de largos tricomas blancos; corona con lóbulos blancos o liláceos, unidos a la columna estaminal a 1,5-2 $\mathrm{mm}$ sobre la base de esta, lateralmente comprimidos, convolutos, de 2-3 $\times 1,5-2,5$ $\mathrm{mm}$, margen superior con un diente corto a cada lado, erecto a suavemente recurvado, ca. 0,5 mm de long.; cabeza estilar exerta o parcialmente oculta por la corona; anteras con alas de 1,8-2 mm de long.; retináculo de $0,4 \times$ $0,15 \mathrm{~mm}$, obovado, caudículas ca. $0,3 \mathrm{~mm}$ de long.; polinios de 1,6 × 0,4 mm, aplanados, asimétricamente lanceolados a falcados. Frutos folículos con pedúnculos contortos, globosos a subglobosos, de 4-7 cm de diám., inflados, pubescentes, cubiertos con apéndices filiformes ca. $1 \mathrm{~cm}$ de long. Semillas comosas, obovoides, de 3,5-5 × 1-25 mm, superficie verrucosa.

Distribución geográfica y hábitat: Especie originaria del sur de Mozambique, Sudáfrica y Suazilandia. Creceen pastizales estacionalmente húmedos y planicies de inundación, desde el nivel del mar hasta ca. $1800 \mathrm{~m}$ de altitud; también en ambientes perturbados y campos de cultivo. Se halla ampliamente introducida y naturalizada en diversas partes de África y otras zonas cálidas del mundo (Goyder et Nicholas, 2001). En Europa (Portugal, España, Italia, Grecia), Australia y los Estados Unidos, se ha indicado como invasora o maleza (Randall, 2017). En Brasil se halla naturalizada en el este del país (Koch et al., 2015). Su cultivo en la Argentina fue señalado por Hurrell et al. (2004). Se menciona como naturalizada en la provincia de Misiones (Zanotti et al., 2020) y se registra por primera vez para las provincias de Entre Ríos (Jozami et Muñoz, 1982) y Buenos Aires y la Ciudad Autónoma de Buenos Aires. Crece en ambientes abiertos, campos de pastoreo y terrenos alterados, y en bordes de rutas asfaltadas y caminos terrados, donde forma poblaciones más o menos densas (Figs. $1,2)$.

Fenología, polinización, dispersión: Florece en primavera-verano, fructifica desde el verano. Se reproduce por semillas y tiene crecimiento rápido; requiere sitios asoleados, suelos sueltos, bien drenados. Es tolerante a los suelos pobres y a las bajas temperaturas. La polinización es entomófila (mayormente realizada por avispas). En Misiones se constató que las flores son visitadas por las avispas neotropicales Agelaia vicina (de Saussure, 1854), Mischocyttarus flavicans (Fabricius, 1804) y Polistes actaeon (Hymenoptera: Vespidae) (Haliday, 1836), que transportan polinias en sus patas (Fig. 2). Además, se ha observado un amplio espectro de visitantes florales, coleópteros y lepidópteros. Las semillas con pelos sedosos tienen dispersión anemócora.

Nombres vernáculos: "Bolas de fraile", "bolitas de gato", "globitos", "planta de la seda" (Argentina), "bolsas de Adán” (Ecuador), "chayote de aire" (Costa Rica), "huevos de gringo" (Honduras).

Usos: Se cultiva como ornamental para parques y jardines. Los frutos se emplean en arreglos florales secos. También se ha cultivado para la obtención de fibras, y por sus usos medicinales: en Kenia se emplea en medicina popular para combatir la demencia (Li et al., 1995; Hurrell et al., 2004). Los frutos son tóxicos para las ovejas (Quattrocchi, 2016).

Material examinado: ARGENTINA. Buenos Aires: Pdo. La Plata, alrededores de La Plata, en jardines y baldíos, 2-VII-1995, Jones s.n. (LP). Ciudad Autónoma de Buenos Aires: Liniers, vereda de calle Rivadavia al 10700, 30-IV-2019, Agnolin \& Agnolin s.n. (LP). Entre Ríos: Dep. Federación, Federación, camino vecinal, 12-XI-1981, Muñoz 1766 (ERA, SI). Dep. La Paz, Termas de La Paz, en campos húmedos, 9-V-2002, Muñoz 6556 (ERA); cerca de Termas de La Paz, campos graminosos bajos, 31-V-2002, Muñoz 6556 (ERA). Misiones: Dep. San Pedro, borde de caminos, 26 25'43,2"S 53 53'24'”W, 26-IX2014, Keller \& Morillo 12280 (CTES, SI).

Material adicional cultivado: ARGENTINA. Ciudad Autónoma de Buenos Aires: Facultad de Agronomía, Universidad de 

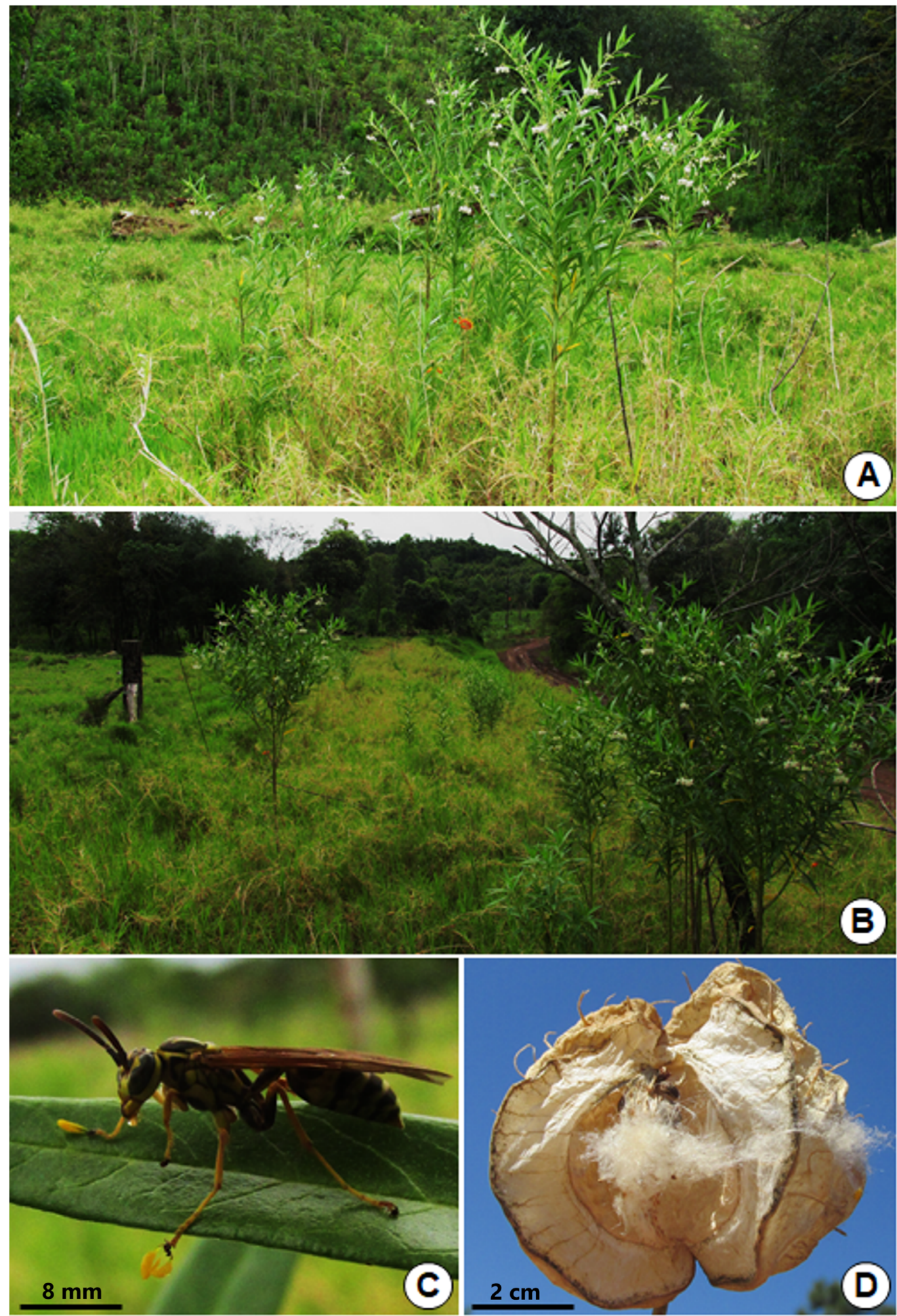

Fig. 1. Gomphocarpus physocarpus en campos de pastoreo y bordes de camino de Misiones. A y B: Poblaciones. C: Avispa Agelaia multipicta con polinios en las patas. D: Fruto en dehiscencia (Fotos de Héctor Keller).

Fig. 1. Gomphocarpus physocarpus populations in pasture fields and roadsides, Misiones. A and B: Populations. C: Agelaia multipicta wasp with pollinia on the legs. D: Fruit in dehiscence (Photos by Héctor Keller). 


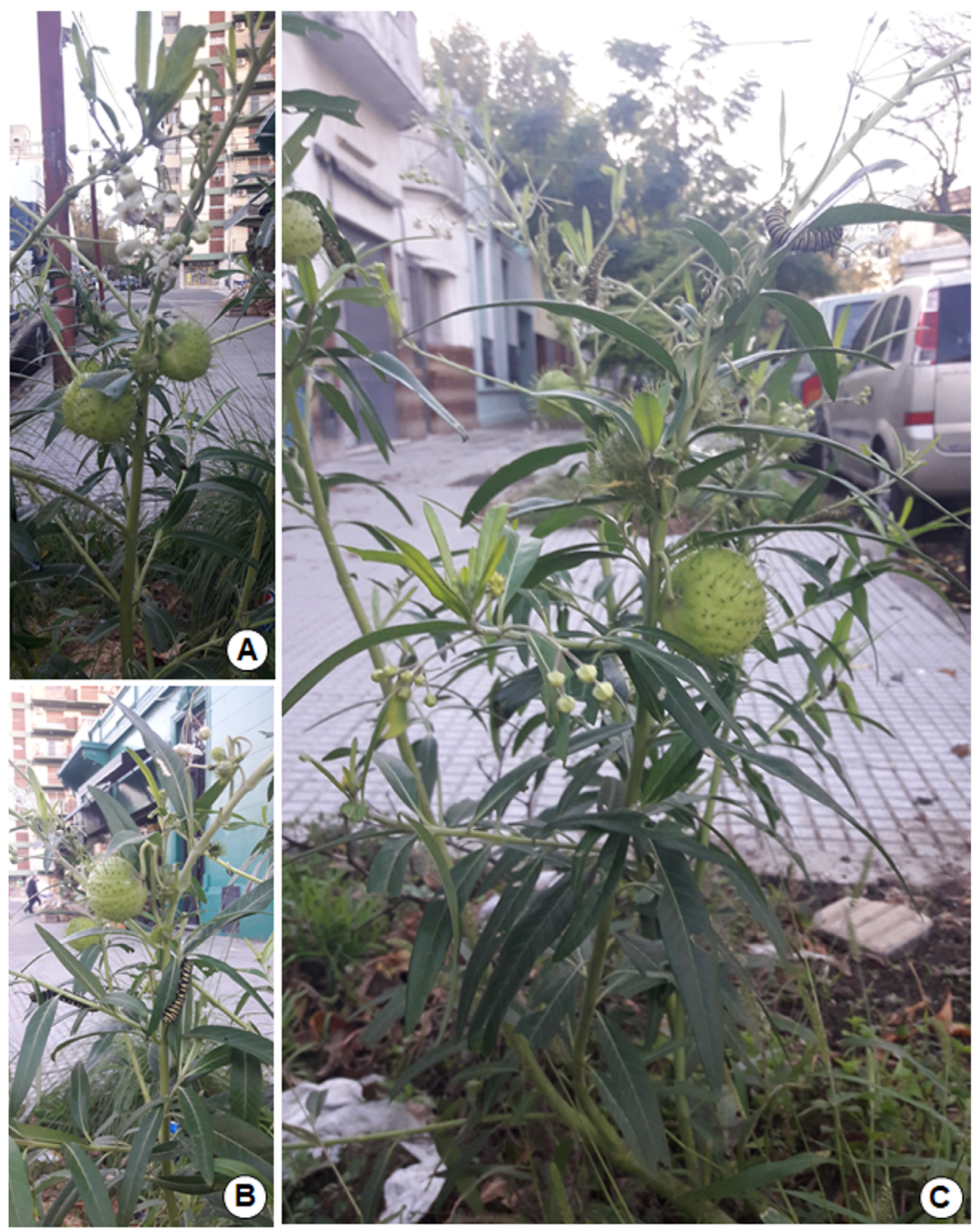

Fig. 2. Gomphocarpus physocarpus en Buenos Aires. A, B, C. Distintos detalles de las plantas halladas. Fig. 2. Gomphocarpus physocarpus in Buenos Aires. A, B, C. Different details of the plants found.

Buenos Aires, en el parque, I-2004, Hurrell \& Bazzano 5422 (SI).

Obs. En la Ciudad Autónoma de Buenos Aires se observó (ejemplar F. Agnolin \& A. Agnolin s.n.) su consumo por parte de orugas de la mariposa "monarca del sur", Danaus erippus (Lepidoptera: Nymphalidae) (Cramer, 1775), y en sus tallos se observaron las hormigas Acromyrmex lundi (Guerin, 1838) y Monomorium pharaonis (Hymenoptera: Formicidae) (Mayr, 1865) y el coccinélido Eriopis connexa (Coleoptera: Coccinellidae) (Germar, 1823). 


\section{Clave de identificación de las especies presentes en la Argentina}

1. Tallos ramificados desde la base. Láminas foliares lineares a linear-elípticas hasta de 1,3 $\mathrm{cm}$ lat. Folículos ovoides con ápice gradual o abruptamente atenuado en un rostro largo.

1. G. fruticosus

1'. Tallos ramificados en la parte superior. Láminas foliares oblongas o angostamente elípticas hasta de $2 \mathrm{~cm}$ lat. Folículos subglobosos, ápice redondeado, sin rostro.

2. G. physocarpus

\section{Discusión y conclusiones}

El proceso de naturalización se inicia con la introducción de una especie exótica que escapa de cultivo y eventualmente deviene naturalizada y potencialmente invasora. Las especies naturalizadas conforman poblaciones que se expanden e incorporan a la flora local sin intervención humana directa, al menos por diez años, y sin comprometer la integridad de las comunidades locales, como es el caso de las invasoras (Rejmánek, 2000; Richardson et al., 2000, 2010, 2011; Pyšek et Richardson, 2006; Randall, 2017; Hurrell et al., 2019). Gomphocarpus fruticosus y $G$. physocarpus se introdujeron en el país como plantas ornamentales, y en el "continuum" del proceso de naturalización se han escapado de cultivo. Hasta el momento, G. fruticosus perdura como escapada de cultivo "ocasional", dado que no llega a naturalizarse. En cambio, G. physocarpus ha logrado colonizar ambientes principalmente abiertos y sitios perturbados, considerándose aquí una especie "naturalizada", que no ha devenido hasta ahora "invasora" como ocurre en otras partes del mundo. La presencia recurrente de $G$. physocarpus en bordes de caminos y de rutas indica una expansión debida a la dispersión anemócora, facilitada por esos "corredores" que constituyen vías de acceso libres del obstáculo que presenta la vegetación local (Trombulak et Frissell, 2000). Este fenómeno se ha observado en Misiones para otras especies naturalizadas, cuyas semillas son transportadas por las corrientes de aire (Hurrell et al., 2019).
La polinización eficiente en pequeñas poblaciones "fundadoras" de G. physocarpus, como resultado de su sistema generalista de polinización por avispas, contribuye al éxito de la colonización y expansión de estas plantas. La presencia de avispas similares en distintas partes del mundo ha facilitado la expansión del rango geográfico y la naturalización de estas plantas (Coombs et al., 2009). Las pequeñas poblaciones de $G$. physocarpus pueden alcanzar altos niveles de éxito de polinización y fructificación. Algunas poblaciones son genéticamente incompatibles y dependen de las avispas para la transferencia del polen entre diferentes plantas. No obstante, algunas poblaciones en Australia, donde esta especie es invasora, parecen ser auto-compatibles. Incluso en esos casos, la polinización cruzada favorece, en general, una mayor producción de frutos (Forster, 1994; Coombs et al., 2009).

\section{Agradecimientos}

Los autores expresan su gratitud al personal de los herbarios CTES (Instituto de Botánica del Nordeste), ERA (Universidad Nacional de Entre Ríos, Facultad de Ciencias Agropecuarias), LP (Plantas Vasculares, Museo de La Plata) y SI (Instituto de Botánica Darwinion). A Daniel H. Bazzano por el material fotográfico aportado, y a los revisores anónimos por sus oportunas sugerencias y comentarios.

\section{Bibliografía}

Coombs, G., Peter, C. I. \& Johnson, S. D. (2009). A test for Allee effects in the self-incompatible wasp-pollinated milkweed Gomphocarpus physocarpus. Austral Ecology 34: 688-697. https:// doi.org/10.1111/j.1442-9993.2009.01976.x

Dematteis, M. \& Ferrucci, M. S. (2017). Las plantas vasculares del Parque Nacional Mburucuyá: Nombres vernáculos y usos. IBONE (UNNECONICET), Corrientes.

Dimitri, M. J. (1988). Asclepias. Enciclopedia Argentina de Agricultura y Jardinería 1: 894-895. Acme, Buenos Aires. 
FABRIS, H. A. (1966). Una asclepiadácea africana cultivada como ornamental: Asclepias fruticosa L. Revista de la Facultad de Agronomía (3ra. época) 42: 75-77.

Fernández Haeger, J. \& Jordano Barbudo, D. (2009). La mariposa monarca Danaus plexippus (L., 1758) en el Estrecho de Gibraltar (Lepidoptera: Danaidae). SHILAP Revista de lepidopterología 37: 421-438.

ForSTER P. I. (1994). Diurnal insects associated with the flowers of Gomphocarpus physocarpus E. Mey. (Asclepiadaceae), an introduced weed in Australia. Biotropica 26: 214-217. https://doi.org/10.2307/2388811.

Fournier, E. (1885). Asclepiadaceae. En Martius, C. F. P. \& A. W. EICHLER (eds.), Flora Brasiliensis 6(4): 189-332. München, Wien, Leipzig.

Goyder, D. J. \& Nicholas, A. (2001). A revision of Gomphocarpus R. Br. (Apocynaceae: Asclepiadeae). Kew Bulletin 56: 769-836. https://doi.org/10.2307/4119297

Hurrell, J.A., Bazzano, D. H. \& Delucchi, G. (2004). Biota Rioplatense IX. Arbustos 2. Nativos y exóticos. Ed. Lola, Buenos Aires.

Hurrell, J. A., Keller, H. A. \& Delucchi G. (2019). Gladiolus dalenii (Iridaceae, Crocoideae) naturalizada en Misiones, Argentina. Bonplandia 28: 89-98. https:// doi.org/10.30972/bon.2823852.

JozAMI, J. M. \& MuÑoz, J. D. (1982). Árboles y arbustos indígenas de la Provincia de Entre Ríos. IPNAYS, CONICET-UNL, Santa Fe.

Koch, I., Rapini, A., Simões, A. O., Kinoshita, L. S., Spina,A. P. \& CAstello, A. C. D. (2015). Apocynaceae. En Lista de Espécies da Flora do Brasil. Jardim Botânico do Rio de Janeiro. Disponible: http://floradobrasil.jbrj.gov.br/ jabot/floradobrasil/FB104792 (Consulta: X-2019).

Li, P. T., Gilbert, M. G. \& Douglas Stevens, W. (1995). Gomphocarpus. En Wu, Z. Y. \& P. H. Raven (eds.), Flora of China 16: 204. Sci. Press, Beijing; Missouri Botanical Garden Press, St. Louis.
PYŠEK, P. \& RichaRdSON, D. M. (2006). The biogeography of naturalization in alien plants. Journal of Biogeography 12: 2040-2050. https://doi.org/10.1111/j.1365-2699.2006.01578.x

Quattrocchi, U. (2016). CRC World Dictionary of Medicinal and Poisonous Plants. CRC Press, Boca Raton.

RANDALL, R. P. (2017). A Global Compendium of Weeds. Tercera Ed. R. P. Randall, Perth, Western Australia.

RejmáneK, M. (2000). Invasive plants: approaches and predictions. Austral Ecology 25: 497-506. https://doi.org/10.1046/j.1442-9993.2000.01080.x

Richardson, D. M., Pyšek, P., Rejmánek, M., Barbour, M. G., Dane Panetta, F. \& West, C. J. (2000). Naturalization and invasion of alien plants: concepts and definitions. Diversity and Distributions 6: 93-107. https://doi.org/10.1046/j.1472-4642.2000.00083.x

Richardson, D. M., Daehler, C. C., Leishman, M. R., PAuchard, A. \& PyšeK, P. (2010). Plant invasions: theoretical and practical challenges. Biological Invasions 12: 3907-3911. https://doi.org/10.1007/s10530-010-9845-1

Richardson, D. M., Pyšek, P. \& CARlton, J. T. (2011). A compendium of essential concepts and terminology in invasion ecology. En Richardson, R. M. (ed.), Fifty Years of Invasion Ecology: The Legacy of Charles Elton, pp. 409-420. Blackwell, Chichester. https://doi.org/10.1002/9781444329988.ch30

Trombulak, S. \& Frissell, C. (2000). Review of ecological effects of roads on terrestrial and aquatic communities. Conservation Biology 14: 18-30. https://doi.org/10.1046/j.1523-1739.2000.99084.x.

Zanotti, C. A., Keller, H. A. \& Zuloaga, F. O. (2020). Vascular flora biodiversity of the province of Misiones, Paranaense region, Argentina. Darwiniana, nueva serie 8: 42-291. https://doi.org/10.14522/darwiniana.2020.81.878. 